\title{
DECISIONS
}

\section{Acute urinary tract infection in infants and young children}

\section{Nader Shaikh MD MPH}

Previously published at www.cmaj.ca

A nine-month-old girl is brought to the family physician's office with a temperature of $39.5^{\circ} \mathrm{C}$ for two days. She has been slightly fussier than usual but has been eating well and acting appropriately. Her vaccinations are up to date. There are no other reported symptoms, and her medical history is clear. On examination, she appears well, and there is no identifiable source of the fever.

\section{Is this a urinary tract infection?}

Yes, it could be. Urinary tract infection should be considered in the differential diagnosis for infants and young children with fever. Several studies have shown that infants with urinary tract infection often present with fever as the only symptom. ${ }^{1,2}$ The probability of infection is high in young children presenting with the factors noted in Box $1 .{ }^{3}$

Ask about a history of urinary tract infection. The duration and height of the fever are important: children with a fever lasting more than 48 hours or with a temperature higher than $39^{\circ} \mathrm{C}$ are at greater risk. ${ }^{3}$

A thorough physical examination should include palpation of the suprapubic area; any tenderness, even in infants, suggests a urinary tract infection. ${ }^{4}$

\section{Is a urine culture necessary?}

Because signs and symptoms are nonspecific in infants, ${ }^{5}$ collect a urine sample and test it using a urine dipstick. With the high rate of false-positive results, a specimen obtained using a perineal bag is helpful only when the result of a urine dipstick test is negative. ${ }^{6}$ If the result is positive (i.e., presence of nitrates or leukocytes), obtain another specimen by catheterization.

If a urine dipstick in a urine specimen obtained by catheterization shows nitrites or leukocytes, the probability of urinary tract infection is high, and antimicrobial treatment should be started.

Reliance on the dipstick test alone, however, will result in about $12 \%$ of cases being missed because of false-negative dipstick test results. ${ }^{7}$ Therefore, all urine specimens should be sent for culture. Growth of more than 50000 colony-forming units of a uropathogen per millilitre of urine from a catheterized specimen indicates urinary tract infection.

\section{Is admission to hospital required?}

Children with urinary tract infection should be admitted to hospital if they are less than two months of age, have evi-

\section{Box 1: Factors increasing likelihood of urinary tract infection in young children ${ }^{3}$}

On history-taking:

- has a fever without an apparent source

- has a fever lasting more than $\mathbf{4 8}$ hours

- has a history of urinary tract infection

- is uncircumcised (male)

On examination:

- has temperature higher than $39^{\circ} \mathrm{C}$

- appears ill

- has tenderness in the suprapubic area

dence of urosepsis (e.g., lethargy, difficulty breathing, decreased appetite), are in an immunocompromised state or are vomiting. However, most infants and young children can be treated as outpatients. ${ }^{8}$

Local drug resistance should be considered when prescribing antibiotics. Because of increased resistance to trimethoprim-sulfamethoxazole, a third-generation cephalosporin may be needed for first-line treatment of children with urinary tract infection associated with fever.

Fever will resolve in most children in 24 to 48 hours with treatment. ${ }^{8}$ If the patient is not responding appropriately to therapy, he or she should be re-evaluated, the sensitivity of organisms to drugs reviewed and admission to hospital considered. A follow-up urine culture is not necessary if the patient's condition improves. ${ }^{8}$

\section{What about routine imaging and prophylaxis for vesicoureteral reflux?}

Because evidence supporting the use of routine imaging to reduce long-term complications of infection (e.g., renal scarring, hypertension, renal failure) is limited, there is controversy about the optimal strategy for imaging.

The rationale for imaging in young children with urinary tract infection is to identify abnormalities of the genitourinary

From the Department of Pediatrics, Children's Hospital of Pittsburgh, Pittsburgh, USA

CMAJ 2010. DOI:10.1503/cmaj.090428 
tract. In approximately $40 \%$ of those with urinary tract infection and fever, voiding cystourethrography will show evidence of vesicoureteral reflux. ${ }^{8}$

Most children with vesicoureteral reflux are treated with prophylactic antimicrobials in the hope of preventing recurring infection. This approach, however, is not based on evidence from randomized controlled clinical trials. Several recent randomized controlled studies have cast doubt on the efficacy of this approach, ${ }^{9-12}$ but there were substantial shortcomings in the studies' methods (lack of blinding and use of bag specimens), thereby limiting the validity and generalizability of the results.

Several ongoing trials are examining the efficacy of prophylactic antimicrobials in preventing recurrence of urinary tract infection that will likely provide the much-needed evidence to inform future management strategies. Preliminary results from two of these studies ${ }^{13,14}$ suggest that prophylaxis may confer modest protection from recurrent symptomatic urinary tract infections, at least in some subgroups of children.

Until the results of these studies are published, physicians should consider the presenting symptoms, history of urinary tract infection, family history of vesicoureteral reflux and the family's preferences to determine the need for imaging and prophylaxis. Children with dilating vesicoureteral reflux (grades IV and V) and those with recurrent urinary tract infections should be referred to a pediatric urologist.

This article has been peer reviewed.

Competing interests: None declared.

\section{REFERENCES}

1. Hoberman A, Chao HP, Keller DM, et al. Prevalence of urinary tract infection in febrile infants. J Pediatr 1993;123:17-23.

2. Roberts KB, Charney E, Sweren RJ. Urinary tract infection in infants with unexplained fever: a collaborative study. J Pediatr 1983;103:864-7.

3. Shaikh N, Morone NE, Lopez J, et al. Does this child have a urinary tract infection? JAMA 2007;298:2895-904.

4. Shaw KN, Gorelick M, McGowan KL, et al. Prevalence of urinary tract infection in febrile young children in the emergency department. Pediatrics 1998; 102:e16.

5. Shaikh N, Morone NE, Bost JE, et al. Prevalence of urinary tract infection in childhood: a meta-analysis. Pediatr Infect Dis $J$ 2008;27:302-8.

6. McGillivray D, Mok E, Mulrooney E, et al. A head-to-head comparison: "cleanvoid" bag versus catheter urinalysis in the diagnosis of urinary tract infection in young children. J Pediatr 2005;147:451-6.

7. Gorelick MH, Shaw KN. Screening tests for urinary tract infection in children: a meta-analysis. Pediatrics 1999;104:e54.

8. Hoberman A, Wald ER, Hickey RW, et al. Oral versus initial intravenous therapy for urinary tract infections in young febrile children [comment]. Pediatrics 1999; 104:79-86.

9. Garin EH, Olavarria F, Garcia Nieto V, et al. Clinical significance of primary vesicoureteral reflux and urinary antibiotic prophylaxis after acute pyelonephritis: a multicenter, randomized, controlled study. Pediatrics 2006;117:626-32.

10. Montini G, Rigon L, Zucchetta P, et al. Prophylaxis after first febrile urinary tract infection in children? A multicenter, randomized, controlled, noninferiority trial Pediatrics 2008;122:1064-71.

11. Pennesi M, Salvatore CM, Peratoner L. Different clinical presentations of pyelonephritis in children with and without vesicoureteral reflux: an Italian multicenter study. Pediatrics 1998;102:1493-4.
12. Roussey-Kesler G, Gadjos V, Idres N, et al. Antibiotic prophylaxis for the prevention of recurrent urinary tract infection in children with low grade vesicoureteral reflux: results from a prospective randomized study. J Urol 2008;179:674-9.

13. Craig J, Hodson E, Reynolds G, et al. A placebo-controlled randomised trial of antibiotics for the prevention of urinary tract infection in children. Washington (DC): American Society of Nephrology; 2008.

14. Hansson S, Stokland E, Jodal U, et al. Preliminary results of the Swedish Reflux Study. In: International Conference on Vesicoureteral Reflux in Children. Göteborg (Sweden): Congrex; 2009.

Decisions is a series that focuses on practical evidence-based approaches to common presentations in primary care. The articles address key decisions that a clinician may encounter during initial assessment. The information presented can usually be covered in a typical primary care appointment. Articles should be no longer than 650 words, should include one box, figure or table and should begin with a very brief description (75 words or less) of the clinical situation. The decisions addressed should be presented in the form of questions. A box providing helpful resources for the patient or physician is encouraged.

\section{In MDD, relief of symptoms is good.}

\section{But is it good enough?}

*Major depressive disorder

Wyeth

C 2010 Wyeth Canada, owner, now a part of Pfizer Inc. Montreal, Canada H4R $1 \mathrm{~J} 6$ 